\title{
PREVALÊNCIA DE SÍNDROME METABÓLICA EM INDIVÍDUOS BRASILEIROS PELOS CRITÉRIOS DE NCEP-ATPIII E IDF
}

\author{
Marcelo Arruda Nakazone*, Anielli Pinheiro, Maria Christiane Valéria Braga Braile, Marcela Augusta de Souza Pinhel, Gisele Firmino \\ de Sousa, Sidney Pinheiro Júnior, Antônio Carlos Brandão, Juan Carlos Yugar toledo, Domingo Marcolino Braile, Dorotéia Rossi \\ Silva Souza \\ Trabalho realizado na Faculdade de Medicina de São José do Rio Preto; no Instituto Domingo Braile de Cardiologia e no Instituto de Cardiologia e Endocrinologia \\ de São José do Rio Preto, SP
}

* Correspondência Molecular, Núcleo de Pesquisa

em Bioquímica e Biologia Molecular, Faculdade de Medicina de São José do Rio Preto

Avenida Brigadeiro Faria Lima 5416 CEP 1509000,

São José do Rio Preto, SP, Brasil. Tel/Fax: +55 11 3201-5864.

naka_med@yahoo.com
Departamento de Biologia

\begin{abstract}
RESUMO
OBjetivos. Analisar perfil bioquímico e caracterizar síndrome metabólica (SM) em pacientes com acompanhamento cardiológico, conforme critérios de NCEP-ATPIII e IDF.

Métodos. Foram estudados 200 pacientes e 140 controles, considerando colesterol total (CT), fração de colesterol de lipoproteína de alta $(H D L c)$, baixa (LDLC) e muito baixa densidade $(V L D L C)$, triglicérides $(T G)$, glicemia de jejum, cintura abdominal e hipertensão arterial sistêmica (HAS). Admitiu-se nível de significância $\mathrm{P}<0,05$.

Resultados. Pacientes apresentaram níveis elevados de glicemia $(103 \pm 31,4 \mathrm{mg} / \mathrm{dL})$ e reduzidos de $\mathrm{HDLc}(48 \pm 13,4 \mathrm{mg} / \mathrm{dL})$ comparado aos controles ( $88 \pm 29,7 \mathrm{mg} / \mathrm{dL}, \mathrm{P}<0,000 \mathrm{l} ; 53 \pm 15,9 \mathrm{mg} / \mathrm{dL}, \mathrm{P}=0,0075$; respectivamente). Controles do sexo

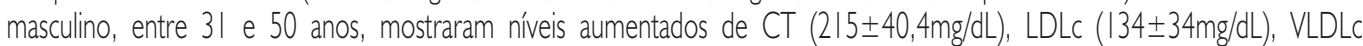
$(30 \pm 1 \mid, 8 \mathrm{mg} / \mathrm{dL})$ e TG $(150 \pm 59,4 \mathrm{mg} / \mathrm{dL})$ comparado às mulheres $(185 \pm 38,2 \mathrm{mg} / \mathrm{dL}, P=0,0137 ; \quad|| \mid \pm 35,8 \mathrm{mg} / \mathrm{dL}$; $P=0,0324 ; 19 \pm 9,7 \mathrm{mg} / \mathrm{dL} ; \mathrm{P}=0,0009 ; 93 \pm 49 \mathrm{mg} / \mathrm{dL}, \mathrm{P}=0,0010 ;$ respectivamente). Mulheres acima dos 50 anos mostraram aumento de CT $(216 \pm 35,9 \mathrm{mg} / \mathrm{dL})$, HDLC $(54 \pm 12,8 \mathrm{mg} / \mathrm{dL})$ e $\mathrm{LDLC}(138 \pm 30,8 \mathrm{mg} / \mathrm{dL})$ comparado aos homens

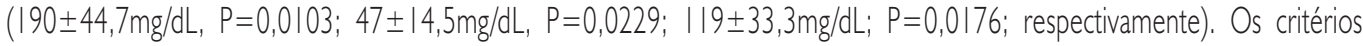
NCEP-ATPIII e IDF caracterizaram SM em 35,5\% e $46 \%$ dos pacientes, respectivamente, com destaque para glicemia, TG e HAS.

Conclusão. Níveis elevados de glicemia e reduzidos de HDLc destacam-se nos pacientes. Perfil lipídico alterado, em homens entre 3 I e 50 anos, confere maior risco para doenças cardiovasculares em adulto jovem, enquanto padrão semelhante nas mulheres, após essa faixa etária, pode refletir alterações hormonais fisiológicas. Ambos os critérios para diagnóstico de SM distinguem pacientes de controles, destacando-se IDF, com parâmetros, no entanto, nem sempre associados a alto risco. Alta prevalência de SM em pacientes, mesmo com acompanhamento cardiológico, sugere predisposiç̧ão para manifestações cardiovasculares em indivíduos brasileiros.
\end{abstract}

Unitermos: Síndrome metabólica. Doenças cardiovasculares. Perfil lipídico. Glicemia. Hipertensão arterial sistêmica.

\section{INTRODUÇÃO}

Há pouco mais de duas décadas, a identificação de um conjunto de fatores de risco para doenças cardiovasculares (DCV), tais como obesidade, hiperglicemia, hipertensão arterial sistêmica (HAS) e dislipidemia visa caracterizar possível predisposição para complicações do aparelho circulatório, como insuficiência cardíaca, doença arterial coronária, infarto agudo do miocárdio e acidente vascular encefálico 1,2. Entretanto, a Organização Mundial da Saúde estabeleceu o termo unificado de síndrome metabólica (SM), considerando os fatores de risco mencionados apenas no final da década de 1990. Em 2001, o "Third Report of the National Cholesterol Education Program Expert Panel on Detection, Evaluation, and Treatment of High Blood Cholesterol in Adults" (NCEP-ATPIII) ${ }^{3}$ definiu critérios clínicos e laboratoriais para o diagnóstico de SM. Recentemente, a "International Diabetes Federation" (IDF) ${ }^{4}$ redefiniu padrões para SM, enfatizando a obesidade central de acordo com o grupo racial do indivíduo.
Desta maneira, considerando sua etiologia multifatorial, sobretudo o fato de geralmente ser desencadeada pela presença de obesidade, sedentarismo, hábitos dietéticos e interação com fatores genéticos, o diagnóstico da SM parece identificar pacientes com risco adicional para diabetes mellitus tipo 2 (DM 2) e DCV, rizados ${ }^{1,5-7}$. Neste sentido, após dados apresentados pelo estudo "Coronary Artery Surgery Study", Nigam et al. ${ }^{8}$ referiram aumento da morbimortalidade em pacientes com SM, ao analisarem banco de dados incluindo, aproximadamente, 25 mil indivíduos com DCV comprovada ou submetidos à cineangiocoronariografia devido à forte suspeita diagnóstica.

Assim, verifica-se atualmente a crescente prevalência de DCV como causa da mortalidade em geral e importante fator desencadeante de incapacidade no Brasil e no mundo 9.10 . Neste contexto, considerando a importância da detecção precoce de SM para a estratificação do risco global de um indivíduo para eventos comparado à análise de fatores de riscos isolados já bem caracte- 
NaKazone MA eT al.

cardiovasculares, este estudo teve como objetivos analisar o perfil bioquímico e caracterizar SM em pacientes com acompanhamento cardiológico e controles, a partir de critérios propostos por NCEPATPIII ${ }^{3}$ e IDF ${ }^{4}$, com o intuito de verificar predisposição para DCV em casuística brasileira.

\section{Métodos}

Foram analisados 340 indivíduos, distribuídos em dois grupos (200 pacientes e 140 controles), pareados por sexo e idade. Foram selecionados como pacientes, indivíduos em acompanhamento regular com médico cardiologista, em um instituto privado, considerando presença de fatores de risco para DCV, com intuito de prevenção primária ou secundária. Foram incluídos no grupo controle indivíduos sem acompanhamento cardiológico de rotina. Todos foram submetidos a exames clínicos e laboratoriais, após concordarem em participar do estudo e assinarem o Termo de Consentimento Livre e Esclarecido.

A análise considerou mensuração de cintura abdominal e investigação de história clínica para DM 2 e HAS, além de parâmetros bioquímicos como glicemia, colesterol total (CT), fração de colesterol de lipoproteína de alta $(H D L c)$, baixa $(L D L C)$ e muito baixa densidade (VLDLC) e triglicérides (TG). Neste caso, os indivíduos foram submetidos à coleta de sangue venoso periférico para análise dos parâmetros laboratorias. Os níveis séricos de TG e CT foram determinados por métodos colorimétricos enzimáticos". O nível de HDLc foi obtido por precipitação seletiva de LDLc e VLDLc com sulfato de dextran na presença de íons $\mathrm{Mg}^{++}$, seguido de dosagem por sistema enzimático colesterol oxidase/peroxidase com colorimetria e leitura, como realizada na dosagem de $\mathrm{CT}^{12}$, em aparelho Cobas Mira S. Os níveis de LDLc e VLDLc foram calculados pela fórmula de Friedewald ${ }^{13}$. O perfil lipídico foi analisado conforme parâmetros propostos pela Sociedade Brasileira de Cardiologia ${ }^{14}$, e a glicemia de jejum conforme normas do Expert Committee on the Diagnosis and Classification of Diabetes Mellitus ${ }^{15}$.

A caracterização de síndrome metabólica considerou cinco parâmetros: circunferência abdominal, níveis de pressão arterial sistêmica e concentrações séricas de glicemia de jejum, TG e de HDLc, conforme duas definições distintas. A primeira, proposta inicialmente pelo NCEP-ATPIII3, em 200I, preconiza o diagnóstico de síndrome metabólica na presença de pelo menos três dos seguintes critérios: cintura abdominal $>88 \mathrm{~cm}$ para mulheres ou $>102 \mathrm{~cm}$ para homens, HDLc $<50 \mathrm{mg} / \mathrm{dL}$ para mulheres ou $<40 \mathrm{mg} / \mathrm{dL}$ para homens, TG $>150 \mathrm{mg} / \mathrm{dL}$, pressão arterial com valores de corte considerando $130 / 85 \mathrm{mmHg}$ e glicemia de jejum $>110 \mathrm{mg} / \mathrm{dL}$. A segunda, proposta pela IDF ${ }^{4}$, em 2005, reduz valores limítrofes para glicemia de jejum ( $\geq 100 \mathrm{mg} / \mathrm{dL})$ e para cintura abdominal ( $\geq 80 \mathrm{~cm}$ para mulheres e $\geq 90 \mathrm{~cm}$ para homens; parâmetros estabelecidos por sul-asiáticos considerando indivíduos das Américas do Sul ou Central).

Os dados coletados foram analisados conforme a prevalência dos fatores de risco isolados ou associados, por meio do teste exato de Fisher para variáveis categóricas, tais como, faixa etária, sexo e critérios para SM, visando estudo comparativo de freqüência entre pacientes e controles. Foram calculados valores médios e desvios padrão para variáveis contínuas com distribuição gaussiana, com utilização do teste $t$ para comparação entre os grupos na avaliação do perfil lipídico ${ }^{16}$. Foi admitido erro $\alpha$ de $5 \%$.

\section{Resultados}

\section{Parâmetros laboratoriais}

Notamos níveis séricos reduzidos de HDLc $(48 \pm 13,4 \mathrm{mg} / \mathrm{dL})$ e elevados de glicemia $(103 \pm 31,4 \mathrm{mg} / \mathrm{dL})$ nos pacientes comparados a controles $(53 \pm 15,9 \mathrm{mg} / \mathrm{dL}, \quad \mathrm{P}=0,0075$ e $88 \pm 29,7 \mathrm{mg} /$ $\mathrm{dL}, \quad \mathrm{P}<0,000 \mathrm{l}$; respectivamente). Níveis séricos elevados de HDLc foram observados nos pacientes do sexo feminino $(5| \pm| 2,8 \mathrm{mg} / \mathrm{dL})$ comparados ao masculino $(45 \pm 13,4 \mathrm{mg} / \mathrm{dL}$, $P=0,0008)$, enquanto nos controles detectaram-se valores aumentados de VLDLc $(25 \pm 13,5 \mathrm{mg} / \mathrm{dL})$ e TG $(133 \pm 87,8 \mathrm{mg} / \mathrm{dL})$, além de reduzidos de HDLc $(49 \pm 15,4 \mathrm{mg} / \mathrm{dL})$ em homens comparados às mulheres $(21 \pm 9,6 \mathrm{mg} / \mathrm{dL}, \quad P=0,0450$; $107 \pm 48,0 \mathrm{mg} / \mathrm{dL}, \quad P=0,0286$ e $57 \pm 15,7 \mathrm{mg} / \mathrm{dL}, \quad P=0,0064$; respectivamente). Ainda, considerando o sexo masculino, notaram-se controles com níveis séricos elevados de $\mathrm{HDLC}$ $(49 \pm 15,4 \mathrm{mg} / \mathrm{dL})$ e reduzidos de glicemia $(88 \pm 19,3 \mathrm{mg} / \mathrm{dL})$ comparados a pacientes $(45 \pm 13,4 \mathrm{mg} / \mathrm{dL}, \quad P=0,0402$ e $100 \pm 21,1 \mathrm{mg} / \mathrm{dL}, \quad \mathrm{P}=0,0020$; respectivamente), enquanto muIheres do grupo controle mostraram valores aumentados de HDLc $(57 \pm 15,7 \mathrm{mg} / \mathrm{dL})$ e reduzidos de TG $(107 \pm 48,0 \mathrm{mg} / \mathrm{dL})$ e glicemia $(89 \pm 38,5 \mathrm{mg} / \mathrm{dL})$ em comparação às pacientes $(5| \pm 12,8 \mathrm{mg} / \mathrm{dL}, \quad P=0,0154 ; \quad| 27 \pm 78,6 \mathrm{mg} / \mathrm{dL}, \quad P=0,0338$ e $105 \pm 37,1 \mathrm{mg} / \mathrm{dL}, \quad \mathrm{P}=0,0065$, respectivamente).

Indivíduos controles acima de 50 anos mostraram níveis séricos aumentados de CT $(202 \pm 43 \mathrm{mg} / \mathrm{dL})$ e LDLC $(127 \pm 33 \mathrm{mg} / \mathrm{dL})$ e reduzidos de VLDLc $(24 \pm 9 \mathrm{mg} / \mathrm{dL})$ e glicemia $(91 \pm 38 \mathrm{mg} / \mathrm{dL})$ comparados aos pacientes $(187 \pm 48 \mathrm{mg} / \mathrm{dL}$, $P=0,0302 ; \quad|| 4 \pm 44 \mathrm{mg} / \mathrm{dL}, \quad P=0,0049 ; \quad 27 \pm 12 \mathrm{mg} / \mathrm{dL}$, $P=0,0304 ; \quad 105 \pm 29 \mathrm{mg} / \mathrm{dL}, \quad P=0,0054 ; \quad$ respectivamente). Também nos controles até a terceira década de vida, observamos no sexo masculino níveis elevados de HDLc comparados aos pacientes ( $P=0,007$ I; Tabela I). Em relação à faixa etária entre 31 e 50 anos, mulheres em acompanhamento médico apresentaram maiores concentrações de LDLc comparadas àquelas do grupo controle $(P=0,0425)$. Por outro lado, no grupo controle, o sexo masculino, na referida faixa etária, mostrou níveis aumentados de CT, LDLc, VLDLc e TG comparados ao sexo feminino $(P=0,0137 ; \quad P=0,0324 ; \quad P=0,0009 ; \quad P=0,0010$, respectivamente). Já a análise de indivíduos acima dos 50 anos evidenciou níveis séricos elevados de HDLc no sexo feminino, comparados ao masculino para pacientes $(P=0,0084)$ e controles $(P=0,0229)$. Além disso, pacientes do sexo masculino apresentaram concentrações aumentadas de VLDLc e glicemia comparadas às mulheres $(P=0,0265 ; P=0,0027$, respectivamente). Nesta mesma faixa etária, notamos no grupo controle, para o sexo feminino, valores aumentados de CT, HDLc e LDLc comparados ao masculino $(P=0,0103 ; P=0,0229 ; \quad P=0,0176$, respectivamente) e ao feminino nos pacientes, considerando níveis de CT $(P=0,0028)$ e LDLC $(P=0,0005)$. 


\begin{tabular}{ccccccccccccccccc}
\hline \multicolumn{10}{c}{ Tabela I - Valores médios e desvios padrão para parâmetros bioquímicos considerando gênero e faixa etária em anos } \\
para pacientes com acompanhamento cardiológico e controles
\end{tabular}

$M=$ média e $D P=$ desvio padrão.

$C T=$ colesterol total, $H D L C=$ fração de colesterol de lipoproteína de alta densidade, LDLC= fração de colesterol de lipoproteína de baixa densidade, VLDLC= fração de colesterol de lipoproteína de muito baixa densidade, $T G=$ triglicérides.

$a \neq b: P=0,0028 ; \quad c \neq c^{\prime}: P=0,0137 ; d \neq d^{\prime}: P=0,0103 ; \quad e \neq f: P=0,0071 ; g \neq g^{\prime}: P=0,0084 ; h \neq h^{\prime}: P=0,0229 ; \quad i \neq 1: P=0,0425 ; \mid \neq m: P=0,0005 ;$

$n \neq n ;: P=0,0324 ; 0 \neq 0$ : $P=0,0176 ; p \neq q: P=0,0265 ; r \neq r^{\prime}: P=0,0009 ; s \neq s^{\prime}: P=0,0010 ; t \neq u: P=0,0027$

\section{Caracterização de síndrome metabólica \\ Conforme critérios do NCEP-ATPIII (200I)}

Esta análise considerou todos os parâmetros para caracterizar o indivíduo como portador de SM, compreendendo no mínimo três e no máximo cinco deles. Evidenciou-se SM em 35,5\% dos pacientes e em 8,6\% dos controles $(\mathrm{P}<0,000 \mathrm{I})$. No caso dos pacientes, 12 deles $(16,9 \%)$ preencheram cinco parâmetros para tal diagnóstico, $20(28,1 \%)$ apresentaram quatro parâmetros e 39 indivíduos (55\%) somaram três parâmetros. Nos controles, nenhum indivíduo com diagnóstico de SM apresentou cinco parâmetros, apenas uma mulher (8,3\%) foi encontrada com quatro critérios, e em II deles $(91,7 \%)$ foram observados três parâmetros-diagnósticos.

A Tabela 2 mostra caracterização de SM, conforme três critérios. Os pacientes do sexo masculino mostraram prevalência de alteração de cintura abdominal $(73,3 \%)$ e no sexo feminino observou-se nível reduzido de HDLc (83,3\%), enquanto valor elevado de pressão arterial destacou-se em ambos os grupos, prevalecendo em mulheres pacientes $(P=0,0070)$. Já em relação aos controles, níveis elevados de TG foram evidenciados em 100\% dos indivíduos, além de cintura abdominal alterada nas mulheres. Níveis elevados de glicemia e pressão arterial prevaleceram nos pacientes comparados aos controles $(P=0,0458 ; P=0,0036$, respectivamente), enquanto nos controles, concentrações séricas aumenta- das de TG $(P<0,000 \mathrm{I})$ destacaram-se como parâmetro-diagnóstico para SM, considerando tanto o sexo masculino $(P=0,0325)$ quanto o feminino $(P=0,0003)$ em relação aos pacientes.

A Tabela 3 mostra a análise de indivíduos que não constituíram critério para diagnóstico de SM. Observou-se aumento da pressão arterial, prevalente nos pacientes $(\mathrm{P}<0,000 \mathrm{I})$, tanto para homens $(4 \mathrm{I}, \mathrm{I} \% ; \quad \mathrm{P}<0,000 \mathrm{I})$, quanto para mulheres $(39,4 \%$; $\mathrm{P}<0,000 \mathrm{I}$ ), comparado aos controles. Por outro lado, nos controles do sexo masculino prevaleceram níveis alterados de TG $(26,8 \%)$, e reduzidos de HDLc nas mulheres (31,6\%). Além disso, notamos dois pacientes (I\%) com caracterização de SM, conforme NCEP-ATPIII, mas não pelos critérios da IDF, e o mesmo ocorreu em dois dos controles $(1,4 \%)$.

\section{Conforme critérios da IDF (2005)}

De acordo com os critérios IDF, evidenciou-se SM em 46\% dos pacientes e em 17,9\% dos controles $(\mathrm{P}<0,000 \mathrm{I})$. Nos pacientes, 16 deles $(17,4 \%)$ obtiveram cinco parâmetros-diagnósticos, 33 (35,9\%) apresentaram quatro parâmetros, enquanto 43 indivíduos $(46,7 \%)$ somaram três parâmetros. Nos controles, nenhum indivíduo portador de SM apresentou cinco parâmetros, três deles (12\%) somaram quatro parâmetros e em 22 deles (88\%) foram constatados três parâmetros.

A análise dos pacientes demonstrou pressão arterial elevada em 


\begin{tabular}{|c|c|c|c|c|c|c|c|c|c|c|}
\hline \multirow[t]{2}{*}{ Grupo } & \multicolumn{2}{|c|}{$\begin{array}{l}\text { Cintura abdominal } \\
\text { alterada }\end{array}$} & \multicolumn{2}{|c|}{$\begin{array}{c}\text { Glicemia de jejum } \\
\text { elevada }\end{array}$} & \multicolumn{2}{|c|}{ HDLcreduzido } & \multicolumn{2}{|c|}{ TG aumentado } & \multicolumn{2}{|c|}{$\begin{array}{c}\text { Pressão arterial } \\
\text { elevada }\end{array}$} \\
\hline & NCEP-ATPIII & IDF & NCEP-ATPIII & IDF & NCEP-ATPIII & IDF & NCEP-ATPIII & IDF & NCEP-ATPIII & IDF \\
\hline \multicolumn{11}{|l|}{$\begin{array}{l}\text { Paciente } \\
\text { Masculino }\end{array}$} \\
\hline N & II & 20 & 10 & 10 & 9 & II & 5 & 5 & 10 & 15 \\
\hline$\%$ & 73,3 & 100,0 & 66,7 & 50,0 & 60,0 & 55,0 & $33,3^{c}$ & $25,0^{8}$ & 66,7 & $75,0^{n}$ \\
\hline$N_{\text {total }}$ & 15 & 20 & 15 & 20 & 15 & 20 & 15 & 20 & 15 & 20 \\
\hline \multicolumn{11}{|l|}{ Feminino } \\
\hline N & 18 & 23 & $\|$ & 14 & 20 & 14 & 5 & 3 & 18 & 16 \\
\hline$\%$ & 75,0 & 100,0 & 45,8 & $60,9^{a}$ & 83,3 & 60,9 & $20,8^{e}$ & $13,0^{i}$ & $75,0^{\prime}$ & $69,6^{\mathrm{P}}$ \\
\hline $\mathrm{N}_{\text {total }}$ & 24 & 23 & 24 & 23 & 24 & 23 & 24 & 23 & 24,4 & 23 \\
\hline \multicolumn{11}{|l|}{ Controle } \\
\hline N & 3 & 14 & | & 8 & 3 & 8 & 4 & 10 & 1 & 2 \\
\hline$\%$ & 75,0 & 100,0 & 25,0 & 57,1 & 75,0 & 57,1 & $100,0^{d}$ & $71,4^{\mathrm{h}}$ & 25,0 & $14,3^{\circ}$ \\
\hline$N_{\text {total }}$ & 4 & 14 & 4 & 14 & 4 & 14 & 4 & 14 & 4 & 14 \\
\hline \multicolumn{11}{|l|}{ Feminino } \\
\hline N & 7 & 8 & I & I & 5 & 6 & 7 & 8 & I & I \\
\hline$\%$ & 100,0 & 100,0 & 14,3 & $12,5^{b}$ & 71,4 & 75,0 & $100,0^{f}$ & 100,0 & $14,4^{\mathrm{m}}$ & $12,5^{9}$ \\
\hline$N_{\text {total }}$ & 7 & 8 & 7 & 8 & 7 & 8 & 7 & 8 & 7 & 8 \\
\hline$P$ & 0,4162 & 1,0000 & 0,0458 & 0,3015 & 1,0000 & 1,0000 & $<0,000 \mid$ & $<0,000$ & 0,0036 & $<0,0001$ \\
\hline
\end{tabular}

$P$ indica valores referentes ao teste $t$.

$\mathrm{HDLC}=$ fração de colesterol de lipoproteína de alta densidade, $T G=$ triglicérides

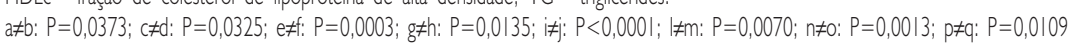

$\mathrm{N}=$ número de indivíduos

destaque nos homens e mulheres (75\% e 69,6\%, respectivamente; Tabela 2). Já nos controles, níveis alterados de TG destacaramse em todas as mulheres. Entre os homens, níveis alterados de TG $(\mathrm{P}<0,000 \mathrm{I})$ prevaleceram nos controles comparados aos pacientes, considerando tanto o sexo masculino $(P=0,0135)$ quanto 0 feminino $(P<0,000 \mathrm{I})$. Por outro lado, observamos níveis pressóricos elevados nos pacientes $(\mathrm{P}<0,000 \mathrm{I})$, para homens $(P=0,00 \mid 3)$ e mulheres $(P=0,0 \mid 09)$, e também glicemia nas mulheres $(P=0,0373)$.

$\mathrm{Na}$ Tabela 3, considerando indivíduos sem critério para SM, verificam-se todos os subgrupos com cintura abdominal alterada, embora sem diferença significante. Em contrapartida, níveis alterados de pressão arterial destacaram-se nos pacientes comparados aos controles $(\mathrm{P}<0,000 \mathrm{I})$, considerando homens e mulheres $(\mathrm{P}<0,000 \mathrm{I})$. Além disso, os critérios da IDF permitiram caracterizar 21 pacientes $(10,5 \%)$ como portadores de SM, além de 13 controles (9,3\%), não diagnosticados pela classificação do NCEP-ATPIII.

\section{Discussão}

Neste estudo, o nível sérico elevado de glicemia destaca-se como fator de risco para doenças cardiovasculares ${ }^{17}$. O perfil lipídico, embora desejável, mostra valores reduzidos de HDLc nos pacientes, sugerindo menor proteção contra aterosclerose nesses indivíduos ${ }^{18-20}$. Apesar disso, valores mais elevados de HDLc são observados nesse grupo no sexo feminino, refletindo padrões estritamente fisiológicos ou influência do uso de anticoncepcionais ou terapia de reposição hormonal ${ }^{21}$.

Considerando adultos até a quinta década de vida, os parâmetros bioquímicos analisados não discriminaram grupos. Entretanto, evidenciam-se níveis limítrofes ou discretamente alterados de CT e LDLc em pacientes e controles, sugerindo aumento da incidência de dislipidemia, inclusive na faixa etária para adulto jovem. Este fato demonstra a necessidade de assistência médica precoce visando a prevenção primária de eventos aterogênicos ${ }^{22}$. Por outro lado, após os 50 anos de idade, estas alterações foram observadas apenas no grupo controle. Tal fato pode refletir indícios de alteração no metabolismo lipídico nestes indivíduos, uma amostra representativa da população em geral nem sempre esclarecida sobre a real composição de uma alimentação saudável ${ }^{23}$, ou desorganizações temporais circadianas, provenientes do envelhecimento do sistema nervoso central24. Ainda, pode sugerir normalização desses parâmetros nos pacientes ora estudados sob intervenção médica, seja com mudança do estilo de vida ou com uso de hipolipemiantes. 


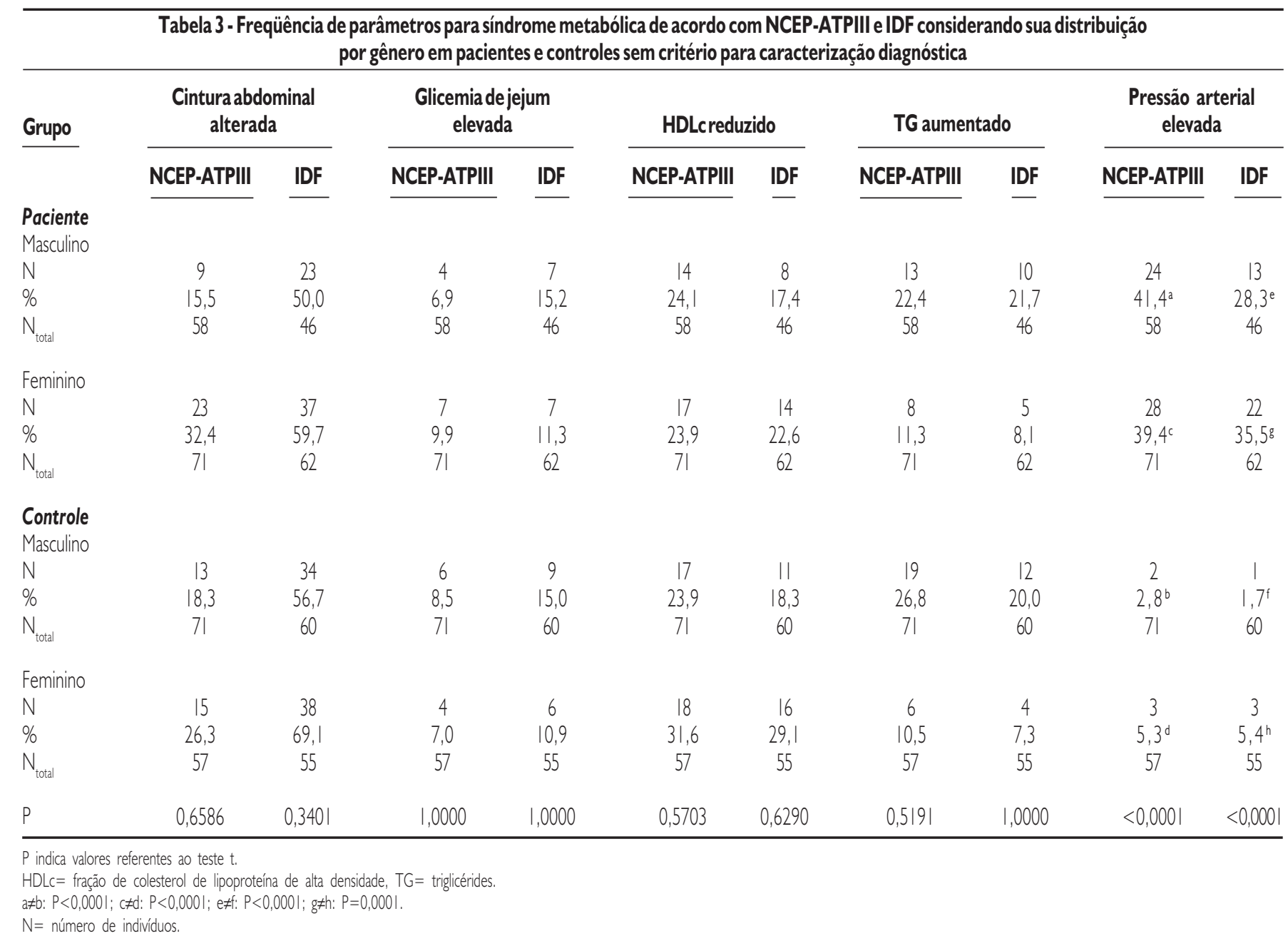

Por outro lado, níveis elevados de glicemia observados nos pacientes, atingindo inclusive valores indesejáveis, confirmam a alta prevalência de resistência insulínica nesses indivíduos com acompanhamento cardiológico, conferindo-lhes caráter de alto risco para doenças cardiovasculares ${ }^{25,26}$. Considerando ainda a faixa etária acima dos 50 anos, a constatação de níveis hiperglicêmicos, principalmente em mulheres, corrobora a análise recente de Legato et al. ${ }^{27}$ em trabalho de revisão sistemática, no qual sugerem acompanhamento criterioso desses casos, considerando risco aumentado para complicações cardiovasculares no sexo feminino.

A análise de ambos os critérios (NCEP-ATPIII e IDF) aplicados para diagnóstico de SM confirma sua capacidade em ampliar a avaliação de um indivíduo, identificando risco cardiovascular adicional tanto em pacientes quanto em controles. Em nossa casuística, incluindo indivíduos brasileiros em acompanhamento regular com médico cardiologista, foram identificados $35,5 \%$ dos pacientes como portadores de SM, conforme NCEP-ATPIII. São dados semelhantes aos recentemente mostrados na população norteamericana, relatando prevalência da síndrome em torno de 34,5 \pm $0,9 \% 28,29$. Comparando esses resultados com estimativas apresen- tadas por Isomaa et al.', em 200I, nessa mesma população, com prevalência de SM entre $21,8 \%$ e 25\%, o que representaria cerca de 47 milhões de pessoas, observamos caráter evolutivo da incidência de fatores predisponentes para DCV.

Nos pacientes, a prevalência de cintura abdominal e níveis pressóricos alterados refletem a elevada incidência de obesidade e HAS em indivíduos de alto risco para DCV ${ }^{30}$. Além disso, níveis hiperglicêmicos conferem efeito adicional para complicações cardiovasculares nesse grupo ${ }^{8}$. Concentrações séricas elevadas de TG nos controles também indicam desvantagem ${ }^{31}$, contribuindo para caracterizar SM em indivíduos previamente isentos de manifestações cardiovasculares. Neste estudo, a constatação da prevalência de HAS em pacientes sem critério para SM alerta para a alta incidência dessa comorbidade como fator de risco independente para DCV 32 .

O diagnóstico de SM, conforme critérios da IDF, caracterizou 46\% dos pacientes em casuística brasileira, corroborando o estudo de Saely et al. ${ }^{33}$, que definiram SM em 45,5\% de casuística austríaca. Porém, ambos os estudos mostraram freqüências de SM superiores a análises envolvendo população norte-americana $(39 \pm 1,2 \%)^{28,29}$ 
NAKAZONE MA ET AL.

e indiana $(25,8 \%)^{34}$. Nesse caso, a valorização da obesidade central pela IDF como parâmetro-diagnóstico, compreendendo valores de referência reduzidos para cintura abdominal comparados ao NCEP-ATPIII, parece justificar o aumento da prevalência da SM ora evidenciado ${ }^{28}$. No entanto, não necessariamente correspondendo maior efeito preditivo de complicações cardiovasculares, conforme demonstrado por Saely et al. ${ }^{33}$. Além disso, a equivalência de padrões antropométricos proposta para populações distintas, vem sendo indicada como possível fator causador de viés nestas análi$\operatorname{ses}^{35}$.

\section{Conclusão}

Níveis séricos elevados de glicemia e reduzidos de HDLC destacam-se nos pacientes. Em controles, o perfil lipídico alterado no sexo masculino entre 31 e 50 anos confere maior risco para DCV no homem adulto jovem, enquanto padrão semelhante evidenciado nas mulheres após essa faixa etária pode refletir apenas alterações hormonais fisiológicas. Ambos os critérios para diagnóstico de SM distinguem pacientes de controles, destacando-se IDF, com parâmetros, no entanto, nem sempre associados a alto risco. A alta prevalência de SM em pacientes, mesmo com acompanhamento cardiológico, sugere predisposição para manifestações cardiovasculares em indivíduos brasileiros.

\section{Agradecimentos}

Às acadêmicas da Faculdade de Medicina de São José do Rio Preto, Fabiana Murakami e Marina Pozo, pela colaboração na coleta dos dados e na seleção dos pacientes, e ao Conselho Nacional de Desenvolvimento Científico e Tecnológico (PIBIC-CNPq 106428/2005-5), pelo apoio financeiro.

\section{Conflito de interesse: não há.}

\section{SUMMARY}

Prevalence of metabolic syndrome using NCEP-ATPIII AND IDF DEFINITIONS IN BRAZILIAN INDIVIDUALS

OBIECTIVES. To analyze the biochemical profile and to characterize metabolic syndrome (MS) in patients with cardiologic medical assistance using NCEP-ATPIII and IDF definitions.

METHODS. Two hundred patients and I 40 controls were studied, considering total cholesterol (TC), HDL-cholesterol (HDLC), $L D L$-cholesterol (LDLC), VLDL-cholesterol $(V L D L C)$, triglycerides (TG), fasting glycemia, abdominal waist and hypertension. Significance level was defined as $P<0.05$.

RESULTS. Patients showed increased glycemia levels $(103 \pm 31.4 \mathrm{mg} / \mathrm{dL})$ and reduced $\mathrm{HDLc}$ levels $(48 \pm 13.4 \mathrm{mg} / \mathrm{dL})$ when compared to controls $(88 \pm 29.7 \mathrm{mg} / \mathrm{dL}, \quad P<0.0001$ and $53 \pm 15.9 \mathrm{mg} / \mathrm{dL}, \quad P=0.0075$; respectively). Male contro/s 3/-50 years old showed increased TC levels $(2 / 5 \pm 40.4 \mathrm{mg} / \mathrm{dL}), L D L$ -

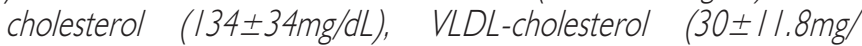
$\mathrm{dL})$ and $T G(150 \pm 59.4 \mathrm{mg} / \mathrm{dL})$ when compared to women $(185 \pm 38.2 \mathrm{mg} / \mathrm{dL}, \quad P=0.0137 ; \quad / / 1 \pm 35.8 \mathrm{mg} / \mathrm{dL} ; \quad P=0.0324 ;$ $19 \pm 9.7 \mathrm{mg} / \mathrm{dL} ; \quad P=0.0009 ; \quad 93 \pm 49 \mathrm{mg} / \mathrm{dL}, \quad P=0.0010$; respectively). Women over 50 years of age showed increased TC concentrations $\quad(216 \pm 35.9 \mathrm{mg} / \mathrm{dL}), \quad H D L$-cholesterol $(54 \pm 12.8 \mathrm{mg} / \mathrm{dL})$ and LDL-cholesterol $\quad(138 \pm 30.8 \mathrm{mg} / \mathrm{dL})$ when compared to men $(190 \pm 44.7 \mathrm{mg} / \mathrm{dL}, \quad P=0.0103$; $47 \pm 14.5 \mathrm{mg} / \mathrm{dL}, \quad P=0.0229 ; \quad 1 / 9 \pm 33.3 \mathrm{mg} / \mathrm{dL} ; \quad P=0.0176 ;$ respectively). NCEP-ATPIII and IDF definitions had characterized MS in $35.5 \%$ and $46 \%$ of patients, respectively, bolding glycemia, TG and hypertension.

CONCLUSION. Elevated glycemia levels and reduced HDLc levels were detected in patients. Altered lipid profile observed in men 3/50 years old signals higher risk for cardiovascular diseases in young adults, while a similar profile in aged women can reflect hormonal physiological changes. Both definitions for MS diagnosis discriminate patients from controls, especially IDF, sometimes with lower capacity to determine high risk for cardiovascular complications. The high prevalence of MS in patients, even with cardiologic medical assistance, suggests predisposition for cardiovascular manifestations in Brazilian individuals. [Rev Assoc Med Bras 2007; 53(5): 407-13]

KEY WORDS: Metabolic syndrome. Cardiovascular disease. Lipid profile. Glycemia. Hypertension.

\section{REFERÊNCIAS}

I. Isomaa B, Almgren P, Tuomi T, Forsen B, Lahti K, Nissen M, et al. Cardiovascular morbidity and mortality associated with the metabolic syndrome. Diabetes Care. 2001;24(4):683-9.

2. Bonaw RO, Smaha LA, Smith Jr SC, Mensah GA, Lenfant C. World Heart Day 2002 - the international burden of cardiovascular disease: responding to the emerging global epidemic. Circulation. 2002; I 06( I 3): | 602-5.

3. The Third Report of the National Cholesterol Education Program (NECP). Expert Panel on Detection. Evaluation, and treatment of high blood cholesterol in adults (Adult Treatment Panel III). JAMA. 200। ; | 6;285( I 9):2486-97.

4. The IDF consensus worldwide definition of metabolic syndrome. Avaliable from: http://www.idf.org

5. Lakka HM, Laaksonen DE, Lakka TA, Niskanen LK, Kumpusalo E, Tuomilehto J, et al. The metabolic syndrome and total and cardiovascular disease mortality in middle-age men. JAMA. 2002;288(21):2709-16.

6. Grundy SM, Hansen B, Smith SC, Cleeman JI, Kahn RA, American Heart Association, et al. Clinical management of metabolic syndrome: report of the American Heart Association/National Heart, Lung, and Blood Institute/American Diabetes Association conference on scientific issues related to management. Circulation. 2004;109(4):55I-6.

7. Miname MH, Chacra APM. Síndrome metabólica. Rev Soc Cardiol Estado de São Paulo. 2005;15(6):477-82.

8. Nigam A, Bourassa MG, Fortier A, Guertin MC, Tardif JC. The metabolic syndrome and its components and the long-term risk of death in patients with coronary heart disease. Am Heart J. 2006; I II (2):5 |421 .

9. Hoyert DL, Kochanek KD, Murphy SL. Deaths: final data for 1997.Natl Vital Stat Rep. 1999;47(19): I-104.

10. World Health Organization. Preventing chronic diseases: a vital investiment. WHO Global Report. Geneva: World Health Organization; 2005.

I I. Bucolo G, David H. Quantitative determination of serum triglycerides by the use of enzymes. Clim Chem. 1973;19(4):476-82.

I 2. Trinder P. Determination of glucose in blood using glucose oxidase with an alternative oxygene acceptor. Ann Clin Biochem. 1969;6(1):24-7. 
13. Friedewald WT, Levy RI, Fredrickson DS. Estimation of the concentration of low-density lipoprotein cholesterol in plasma, without use of the preparative ultracentrifuge. Clin Chem. 1972;|8(6):499502 .

14. Santos RD, Sociedade Brasileira de Cardiologia. III Diretrizes Brasileiras sobre Dislipidemias e Diretriz de Prevenção da Aterosclerose do Departamento de Aterosclerose da Sociedade Brasileira de Cardiologia. Arq Bras Cardiol. 2001;77(3):1-48.

15. The Committee on the Diagnosis and Classification of Diabetes Mellitus. Report of The Expert Committee on the Diagnosis and Classification of Diabetes Mellitus. Diabetes Care. 1997;20(7): I I8397.

16. Montgomery DC. Design and analysis of experiments. $3^{\text {rd }}$ ed. New York: John Wiley \& Sons; 1991.

17. Rasouli M, Kiasari AM. Interactions of serum hsCRP with apoB, apoB/ $\mathrm{Al}$ ratio and some components of metabolic syndrome amplify the predictive values for coronary artery disease. Clin Biochem. 2006;39( I 0):97|-7

18. Franceschini G. Epidemiologic evidence for high-density lipoprotein cholesterol as a risk factor for coronary artery disease. Am J Cardiol. 200।;88(I 2A):9N-I3N

19. Shah PK, Kaul S, Nilsson J, Cercek B. Exploiting the vascular protective effects of high-density lipoprotein and its apolipoproteins: an idea whose time for testing is coming, part I. Circulation. 200 I; I 04( I 9):2376-83.

20. Kuvin JT, Karas RH. The effects of LDL reduction and HDL augmentation on physiologic and inflammatory markers. Curr Opin Cardiol. 2003; I 8(4):295-300.

21. Fisberg RM, Stella RH, Morimoto JM, Pasquali LS, Phillipi ST, Latorre MRDO. Lipid profile of nutrition students and its association with cardiovascular disease risk factors. Arq Bras Cardiol. 2001;76(2): 137-47.

22. Imamura H, Izawa A, Kai R, Yokoseki O, Uchikawa S, Yazaki Y, et al Trends over the last 20 years in the clinical background of young Japanese patients with coronary artery disease. Circ J. 2004;68(3): | 86-9|

23. Margetts BM, Martinez JA, Saba A, Holm L, Kearney M, Moles A. Definitions of 'healthy' eating: a pan-EU survey of consumer attitudes to food, nutrition and health. Eur J Clin Nutr. 1997;5 I (2):23-9.

24. Troti R, Rondanelli M, Cuzzoni G, Ferrari E, D'Eril GM. Circadian temporal organization of lipidic fractions in elderly people. Entrainment to the dietary schedule. Aging Clin Exp Res. 2002; I 4(2):94-9.

25. Miller TD, Redberg RF, Wackers FJ. Screening asymptomatic diabetes patients for coronary artery disease: why not? J Am Coll Cardiol. 2006;48(4):761-4.
26. Rosano GM, Vitale C, Fragasso G. Metabolic therapy for patients with diabetes mellitus and coronary artery disease. Am J Cardiol. 2006;98(5A): | 4J- | 8|.

27. Legato MJ, Gelzer A, Goland R, Ebner SA, Rajan S, Villagra V, et al. Gender-specific care of the patients with diabetes: review and recommendations. Gend Med. 2006;3(2):131-58.

28. Ford ES. Prevalence of the metabolic syndrome defined by the International Diabetes Federation among adults in the U.S. Diabetes Care. 2005;28(II):2745-9.

29. Reynolds K, He J. Epidemiology of the metabolic syndrome. Am J Med Sci. 2005;330(6):273-9.

30. Hurrell C, Wietlisbach $\vee$, Jotterand $V$, Volet $M$, Lenain $V$, Nicod P, et al. High prevalence of major cardiovascular risk factors in first-degree relatives of individuals with familial premature coronary artery diseaseThe GENECARD project. Atherosclerosis.; 2006. [in press]

31. Bujo H, Takahashi K, Saito Y, Maruyama T, Yamashita S, Matsuzawa $Y$, et al. Clinical features of familial hypercholesterolemia in Japan in a database from 1996-1998 by the research committee of the ministry of health, labour and welfare of Japan. J Atheroscler Thromb. 2004; I I (3): | 46-5 |

32. Yologlu S, Sezgin AT, Sezgin N, Ozdemir R, Yesilada E, Topal E. Determination of risk factors in obese and non-obese patients with coronary artery disease. Acta Cardiol. 2005;60(6):625-9.

33. Saely CH, Koch L, Schmid F, Marte T, Aczel S, Langer P, et al. Adult treatment Panel III 200 I but not International Diabetes Federation 2005 criteria of the metabolic syndrome predict clinical cardiovascular events in subjects who underwent coronary angiography. Diabetes Care. 2006;29(4):901-7.

34. Deepa M, Farooq S, Datta M, Deppa R, Mohan V. Prevalence of metabolic syndrome using WHO, ATPIII and IDF definitions in Asian Indians: the Chennai Urban Rural Epidemiology Study (CURES-34). Diabetes Metab Res Rev. 2007;23(2): 127-34.

35. Misra A, Wasir JS, Vikram NK. Waist circumference criteria for the diagnosis of abdominal obesity are not applicable uniformly to all populations and ethnic groups. Nutrition. 2005;2I(9):969-76. 